\title{
Combination of Standard Model and beyond Standard model Higgs searches at DØ
}

\author{
Michael Mulhearn*广 \\ University of Virginia \\ E-mail: mulhearnefnal.gov
}

A combination of searches for Standard Model and beyond Standard Model Higgs boson production at $\mathrm{D} \emptyset$ using a data sample up to $7 \mathrm{fb}^{-1}$ of integrated luminosity is performed. For Standard Model searches we determine combined upper 95\% CL upper limits on the ratio of the Higgs boson cross section times the branching ratio to its Standard Model prediction for Higgs boson masses between 100 and $200 \mathrm{GeV} / \mathrm{c}^{2}$.

35th International Conference of High Energy Physics - ICHEP2010,

July 22-28, 2010

Paris France

\footnotetext{
*Speaker.

†n behalf of the DØ Collaboration.
} 


\section{Introduction}

In the standard model (SM), the spontaneous breakdown of the electroweak symmetry generates masses for the $W$ and $Z$ bosons and produces a scalar massive particle, the Higgs boson, which has so far eluded detection. The mass of the Higgs $m_{H}$ is unknown, but indirect constraints from electroweak measurements and direct searches for the Higgs boson [1] suggest that the value is most likely between 100 and $200 \mathrm{GeV}$ the region currently probed at the Fermilab Tevatron.

In this proceeding, we combine the results of direct searches for SM Higgs bosons in $p \bar{p}$ collisions at $\sqrt{s}=1.96 \mathrm{TeV}$ recorded by the $\mathrm{D} \emptyset$ experiment [2]. The combination [3] includes searches for Higgs bosons produced through gluon-gluon fusion (GGF, $g g \rightarrow H$ ), vector boson fusion (VBF, $\left.q \bar{q} \rightarrow q^{\prime} \bar{q}^{\prime} H\right)$, and in association with vector bosons $(q \bar{q} \rightarrow V H)$. The analyses use data corresponding to integrated luminosities ranging from 2.1 to $6.7 \mathrm{fb}^{-1}$, collected from 2002 to 2010 . The primary Higgs boson decay modes examined are $H \rightarrow b \bar{b}, H \rightarrow W^{+} W^{-}, H \rightarrow \tau^{+} \tau^{-}$and $H \rightarrow \gamma \gamma$. The searches are organized into analysis subsets comprising different Higgs production and decay modes, each designed to isolate a particular final state. The analyses were designed to be mutually exclusive after the event selections.

\section{Analyses Used in the Combination}

For $m_{H}<135 \mathrm{GeV}$ the primary Higgs decay is $H \rightarrow b \bar{b}$. The GGF and VBF production channels are not considered for this decay mode, as the fully hadronic final state suffers from prodigious background at the Tevatron. But the associated production channels $W H \rightarrow \ell v b \bar{b}, Z H \rightarrow \nu \bar{v} b \bar{b}$ and $Z H \rightarrow \ell \ell b \bar{b}$, where $\ell=e, \mu$, have reconstructed leptons or missing transverse energy in the final state and therefore smaller backgrounds. To distinguish the decay $H \rightarrow b \bar{b}$ from background processes involving light quarks or gluons, jets are identified as likely containing $b$-quarks ( $b$-tagged) if they pass loose or tight requirements on the output of a multivariate analysis treated to separate $b$-jets from light-jets [4]. The candiate events are classified as double-tagged (DT) if at least two jets are $b$-tagged and single-tagged (ST) if only one jet is $b$-tagged. For these analyses, each lepton flavor of the $V$ boson decay $(\ell=e, \mu)$ is treated as an independent channel. Additional sensitivity is obtained from the $t \bar{t} H \rightarrow t \bar{t} b \bar{b}$ channel which examines events with up to three $b$-tagged jets.

For $m_{H}>135 \mathrm{GeV}$ the primary Higgs decay is $H \rightarrow W^{+} W^{-}$. In this decay mode, the GGF and VBF production mechanism have resonable background when at least one of the $W$ bosons decays leptonically. We consider three final states of opposite-signed leptons: $W W \rightarrow e^{+} v e^{-} v, e^{ \pm} v \mu^{\mp} v$, and $\mu^{+} v \mu^{-} v$. The $H \rightarrow e^{ \pm} v \mu^{\mp} v$ analysis further separates events in three final states with 0 jets, 1 jet, and two or more jets. A separate analysis considers the $H \rightarrow W^{+} W^{-} \rightarrow \ell v q \bar{q}$ process. In all $H \rightarrow W^{+} W^{-}$decays with $m_{H}<2 M_{W}$, at least one of the $W$ bosons will be off mass shell. There is a small contribution from $H \rightarrow Z Z$ decays, particularly in the $H \rightarrow W^{+} W^{-} \rightarrow e e / \mu \mu v v$ searches. In all cases, lepton selections include both electrons and muons $(\ell=e, \mu)$, while $\tau$ leptons are included in the simulation and the selections have acceptance for secondary leptons from $\tau \rightarrow e / \mu$ decays. For $V H \rightarrow V W^{+} W^{-}$production, we search for leptonic $V$ boson decays with three final states of same-signed leptons: $V W W \rightarrow e^{ \pm} e^{ \pm}+X, e^{ \pm} \mu^{ \pm}+X$, and $\mu^{ \pm} \mu^{ \pm}+X$.

The combined senstivity is improved by including searches optimized for additional Higgs boson decay modes. The $X+H \rightarrow \tau \tau b \bar{b} / q \bar{q} \tau \tau$ analysis selects the $\tau \tau$ plus dijet final state with one 
Table 1: List of analysis channels and corresponding integrated luminosity $L$. In cases where sub-channels use different datasets, a range of integrated luminosities is presented.

\begin{tabular}{|ll|ll|}
\hline Channel & $L\left(\mathrm{fb}^{-1}\right)$ & Channel & $L\left(\mathrm{fb}^{-1}\right)$ \\
\hline$W H \rightarrow \ell v b \bar{b}$, & 5.3 & $H \rightarrow W^{+} W^{-} \rightarrow e v \mu \nu$, & 6.7 \\
$Z H \rightarrow v \bar{v} b \bar{b}$, & $5.2-6.4$ & $H \rightarrow W^{+} W^{-} \rightarrow e e / \mu \mu \nu v$ & 5.4 \\
$Z H \rightarrow \ell \ell b \bar{b}$, & $4.2-6.2$ & $H \rightarrow W^{+} W^{-} \rightarrow \ell v q \bar{q}$ & 5.4 \\
$H \rightarrow \gamma \gamma$ & 4.2 & $V H \rightarrow V W^{+} W^{-}$ & 5.3 \\
$t \bar{t} H \rightarrow t \bar{t} b \bar{b}$ & 2.1 & $X+H \rightarrow \tau \tau b \bar{b} / q \bar{q} \tau \tau$ & 4.9 \\
\hline
\end{tabular}

$\tau$ decaying to $\mu$ and the other decaying hadronically. This analysis is sensitive to $Z H \rightarrow \tau \tau b \bar{b}$, $V H \rightarrow q \bar{q} \tau \tau$, GGF and VBF. The $H \rightarrow \gamma \gamma$ analysis includes signal contributions from GGF, VBF, and associated production.

The analyses used in this combination are listed in Table 1. Since the previous DØ SM Higgs combination [5], we have updated the $W H \rightarrow \ell v b \bar{b}, Z H \rightarrow v v b b, Z H \rightarrow \ell \ell b \bar{b}, V H \rightarrow V W^{+} W^{-}$, and $H \rightarrow W^{+} W^{-} \rightarrow e v \mu v$ analyses. The $H \rightarrow W^{+} W^{-} \rightarrow \ell v q \bar{q}$ channel is a new addition to the combination.

\section{Final Discriminants and Systematic Uncertainties}

The $H \rightarrow \gamma \gamma$ channel uses the diphoton mass as the final discriminant used to search for a Higgs signal, while the $t t H$ channel uses the scalar sum of the transverse momentum from the leading jets in the event. For all other channels, multivariate analyses are trained to discriminate signal from background, with the output distributions taken as the final discriminant. Systematic uncertainties are quantified by their effect on the final discriminant.

The systematic uncertainties differ between analyses for both the signals and backgrounds. Here we summarize only the largest contributions. Most analyses carry an uncertainty on the integrated luminosity of 6.1\% [6], but when possible the overall normalization is instead determined from the NNLO $Z / \gamma^{*}$ cross section using data events near the peak of $Z \rightarrow \ell \ell$ decays. The $H \rightarrow b \bar{b}$ analyses have an uncertainty on the $b$-tagging rate of 1-9\%. These analyses also have an uncertainty on the jet measurement and acceptances of $\sim 7 \%$. All analyses include uncertainties associated with lepton measurement and acceptances, which range from 1-5\% per lepton in the final state. The largest contribution for all analyses is the uncertainty on the background cross sections at 6-30\% depending on the analysis channel and specific background. These values include both the uncertainties on the theoretical cross section calculations and the uncertainties on the higher order correction factors. The uncertainty on the expected multijet background is dominated by the statistics of the data sample from which it is estimated, and is considered separately from the other cross section uncertainties. Several analyses incorporate shape-dependent uncertainties on the kinematics of the dominant backgrounds, derived from the potential deformations of the final variables due to generator and background modeling uncertainties. Further details on the systematic uncertainties are provided in Ref. [3]. 


\section{The SM Higgs Combination}

The outcome of an experiment is more consistent with either the signal-plus-background $(\mathrm{S}+\mathrm{B})$ hypothesis or the background-only (B) hypothesis as quantified by the ratio of the Poisson likelihoods for each hypothesis. Distributions for the log likelihood ratio (LLR) are obtained by generating Poisson fluctuations of the $\mathrm{B}$ and $\mathrm{S}+\mathrm{B}$ hypotheses. By integratring these LLR distributions up to the LLR for the experiment, we determine the confidence intervals $C L_{B}$ and $C L_{S+B}$. Limits are set by adjusting the signal cross section until $C L_{S}=C L_{S+B} / C L_{B}=1-\alpha$, with a $95 \%$ CL limit obtained for $\alpha=0.95$ [7].

All systematic uncertainties originating from a common source are treated as correlated in the combination. For the background rate, they are generally comparable to the signal expectation, so the treatment of systematics is an important part of the limit calculation. The systematic uncertainties are included as nuisance parameters which adjust the $\mathrm{B}$ and $\mathrm{S}+\mathrm{B}$ predictions. To minimize their impact on the sensitivity, the likelihood of the B and S+B hypotheses used to calculate each LLR value are first maximized by independent fits of the nuisance parameters.

As no significant signal-like excess is observed, we derive limits on the SM Higgs boson production $\sigma \times B(H \rightarrow X)$. To accomodate diverse contributions from processes with different cross-sections and sensitivities, we present our results in terms of the ratio of $95 \%$ CL upper cross section limits to the SM predicted cross section as a function of Higgs boson mass. The SM prediction for Higgs boson production would be considered excluded at 95\% CL when this limit ratio falls below unity. Figure 1 shows the expected and observed 95\% CL cross section limit as a ratio to the $\mathrm{SM}$ cross section in the probed mass region $\left(100 \leq m_{H} \leq 200 \mathrm{GeV}\right)$, with all analyses combined. These results are also summarized in Table 2. The expected and observed LLRs are shown in Fig. 1.

Table 2: Combined 95\% CL upper limits on $\sigma \times B(H \rightarrow X)$ for SM Higgs boson production. The limits are reported in units of the SM production cross section times branching fraction.

\begin{tabular}{lccccccccccc}
$m_{H}(\mathrm{GeV})$ & 100 & 105 & 110 & 115 & 120 & 125 & 130 & 135 & 140 & 145 & 150 \\
\hline Expected: & 1.80 & 1.86 & 2.13 & 2.31 & 2.60 & 2.67 & 2.82 & 2.59 & 2.40 & 2.19 & 1.87 \\
Observed: & 1.40 & 1.69 & 1.44 & 2.65 & 3.50 & 4.16 & 3.16 & 4.17 & 3.53 & 3.29 & 2.43 \\
\hline
\end{tabular}

\begin{tabular}{lcccccccccc}
$m_{H}(\mathrm{GeV})$ & 155 & 160 & 165 & 170 & 175 & 180 & 185 & 190 & 195 & 200 \\
\hline Expected: & 1.62 & 1.21 & 1.14 & 1.36 & 1.60 & 1.92 & 2.40 & 2.93 & 3.40 & 3.96 \\
Observed: & 1.93 & 1.17 & 1.03 & 1.10 & 1.35 & 1.86 & 2.86 & 3.27 & 4.44 & 4.97
\end{tabular}

\section{The MSSM Higgs Combination}

In the Minimally Supersymmetric Standard Model (MSSM) the coupling of a degenerate neutral Higgs boson $(\phi)$ to charged leptons and down type quarks is enhanced by a factor $\tan \beta$. Due to their higher mass, the most significant processes involve $b$-quarks, or $\tau$ leptons, which themselves decay hadronically $\left(\tau_{j}\right)$, or to an electron or muon and neutrinos $\left(\tau_{e}\right.$ or $\left.\tau_{\mu}\right)$. The D0 MSSM Higgs combination combines contributions from the $\phi \rightarrow \tau_{j} \tau_{\mu}, \phi \rightarrow \tau_{j} \tau_{e}, \phi \rightarrow \tau_{e} \tau_{\mu}, b \phi \rightarrow b b b$, 


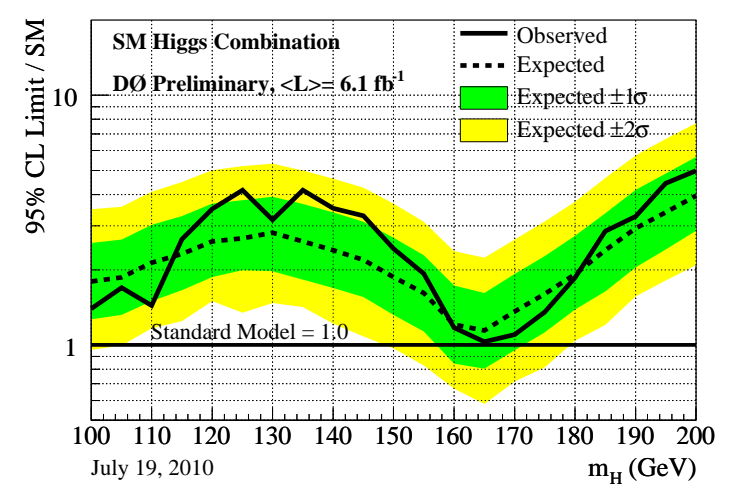

(a)

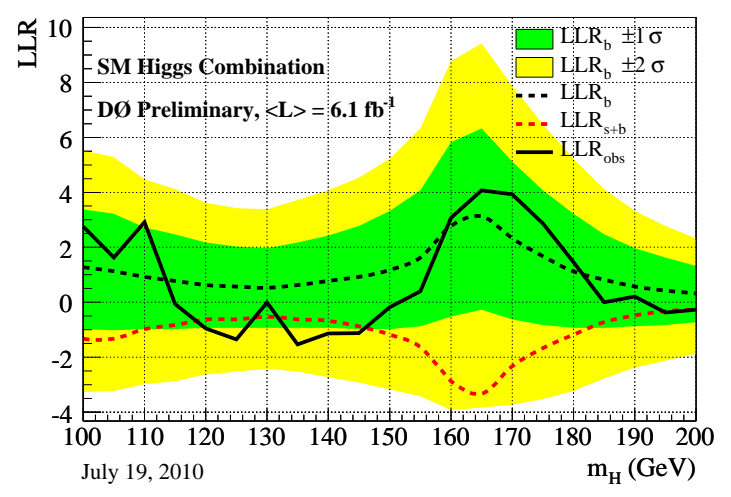

(b)

Figure 1: Expected (median) and observed 95\% CL cross section upper limit ratios (a) for the combined analyses as a function of $m_{H}$. Log-likelihood ratio distribution (b) for the combined analyses as a function of $m_{H}$. The bands indicate the $68 \%$ and $95 \%$ probability regions where the limits can fluctuate in the absence of signal.

and $b \phi \rightarrow b \tau_{j} \tau_{\mu}$ processes using 1.2-2.6 $\mathrm{fb}^{-1}$ of collisions. Limits in four different benchmark scenarios within the MSSM are considered and lower limits on $\tan \beta$ reach values of 30 at the most sensitive mass point [8]

\section{Acknowledgments}

We thank the staffs at Fermilab and collaborating institutions, and acknowledge support from the DOE and NSF (USA); CEA and CNRS/IN2P3 (France); FASI, Rosatom and RFBR (Russia); CNPq, FAPERJ, FAPESP and FUNDUNESP (Brazil); DAE and DST (India); Colciencias (Colombia); CONACyT (Mexico); KRF and KOSEF (Korea); CONICET and UBACyT (Argentina); FOM (The Netherlands); STFC and the Royal Society (United Kingdom); MSMT and GACR (Czech Republic); CRC Program and NSERC (Canada); BMBF and DFG (Germany); SFI (Ireland); The Swedish Research Council (Sweden); and CAS and CNSF (China).

\section{References}

[1] R. Barate et al., Phys. Lett. B 565, 61 (2003); The LEP Electroweak Working Group, http: / / lepewwg.web.cern. ch/LEPEWWG/.

[2] DØ Collaboration, V. Abazov et. al., Nucl. Instrum. Meth. A 565, 463 (2006).

[3] DØ Collaboration, DØ Note 6094-CONF.

[4] T. Scanlon, FERMILAB-THESIS-2006-43.

[5] DØ Collaboration, D $\varnothing$ Note 6008-CONF.

[6] T. Andeen et al., FERMILAB-TM-2365.

[7] W. Fisher, FERMILAB-TM-2386-E.

[8] DØ Collaboration, DØ Note 5935-CONF. 